\title{
Jóvenes de hoy, adolescentes de ayer en Uruguay: maternidad y desempeños ${ }^{1}$
}

\author{
Youth of Today, Teenagers of Yesterday in \\ Uruguay: Maternity and Performances
}

\section{Jovens de hoje, adolescentes de ontem no Uruguai: maternidades y desempenhos}

\section{Carmen Varela Petito ${ }^{2}$}

Profesora de la Universidad de la República, Montevideo, Uruguay

varelapetito@gmail.com

\section{Cecilia Lara ${ }^{3}$}

Asistente de investigación de la Universidad de la República, Montevideo, Uruguay cecilara@gmail.com

1 El trabajo es un producto de la investigación realizada en 2010-2011 con base en los datos recogidos en las Encuestas Nacionales de la Juventud (ENA) 1990 y 2008. El financiamiento proviene de la Facultad de Ciencias Sociales de la Universidad de la República, Uruguay.

2 Diplomatura de Especialización en Demografía.

3 Magíster en Demografía y Estudios de Población. 


\title{
Resumen
}

Este trabajo analiza los diferentes desempeños sociales a los que arriban las jóvenes uruguayas de dos cohortes, que cursan la última etapa de la juventud en 1990 y 2008. Se realiza una diferenciación entre quienes fueron madres en la adolescencia y aquellas que no lo fueron. Se consideran las divergencias entre unas y otras en: paridez alcanzada, educación, inserción laboral, pobreza y reclusión en el ámbito doméstico. También se indaga la interrelación del momento en que se tiene el primer hijo con otros eventos del tránsito a la vida adulta. El enfoque teórico-metodológico se basa en el curso de vida. Los principales resultados revelan que las madres adolescentes arriban a la vida adulta con precariedades que limitan su inserción social.

Palabras clave: maternidad; adolescencia; curso de vida; divergencia social.

\begin{abstract}
This paper studies the social performance of two cohorts of Uruguayan young women, which completed the last youth stage in 1990 and 2008. It compares between teenage mothers and those that were not. The differences among both groups are analyzed considering the gaps in: parity achieved, education, labor market, poverty and home confinement. Besides, the interrelationship of the first birth event with other events related to the transition to adulthood is inquired. The theoretical-methodological approach is based on the course of a lifetime. The main results reveal that the teenage mothers arrive to adulthood with scarcities which limit their social inclusion.
\end{abstract}

Keywords: maternity; adolescence; life course; social divergence.

\section{Resumo}

Este trabalho analisa os diferentes desempenhos sociais experimentados pelas jovens uruguaias de duas coortes, que estão na última etapa da juventude, em 1990 e 2008. Realiza-se uma diferenciação entre aquelas que foram mães na adolescência e aquelas não foram tendo em vista: partos alcançados, educação, inserção laboral, pobreza e permanência no âmbito doméstico. Também se indaga na relação entre o momento em que se tem o primeiro filho com outros eventos do trânsito à vida adulta. O enfoque teóricometodológico baseia-se no curso de vida. Os principais resultados revelam que as mães adolescentes chegam à vida adulta com precariedades que limitam sua inserção social.

Palavras-chave: maternidade, adolescência, curso de vida, divergência social. 


\section{Introducción}

La producción sociodemográfica sobre la fecundidad y la maternidad en la adolescencia en Uruguay ha puesto en evidencia la vinculación de este fenómeno con la desigualdad social, las carencias en salud sexual y reproductiva y las desigualdades de género. También se han mostrado las dificultades de estas adolescentes para realizar otras actividades más allá de la maternidad y la vida doméstica (Varela 1999; Amorín, Carril y Varela 2006; Varela y Fostik 2011). Sin embargo, había quedado pendiente para futuras investigaciones estudiar los desempeños a los que arriban estas jóvenes tanto en el ámbito educativo y laboral como de la vida pública en general, una vez que están próximas a finalizar la juventud y a ingresar a la adultez. Este trabajo ha permitido avanzar en este sentido. Es así como el enfoque teórico-metodológico de esta investigación se basa en el curso de vida y estudia la maternidad adolescente a través del análisis descriptivo de variables que dan cuenta de los desempeños y transiciones de las jóvenes, vinculados con la etapa del ciclo de vida en que iniciaron la reproducción. Para ello se aplica el método Kaplan Meier y un método econométrico probit.

Los principales resultados revelan que las jóvenes que tuvieron su primer hijo en la adolescencia, al final de la juventud, mayoritariamente tienen una paridez elevada (orden 2 o superior), alcanzan educación primaria (seis años en total), presentan baja inserción en el mercado de empleo, se encuentran recluidas en el ámbito doméstico y en situación de pobreza. Este escenario revela que estas jóvenes arriban a la vida adulta con precariedades que limitan su adecuada inserción social y desempeño en ella. La situación es marcadamente diferente con las jóvenes que posponen la maternidad para otras etapas de la juventud o que incluso no pasan por este evento. El momento en que se procesan los distintos eventos de la transición a la adultez, así como los desempeños sociales, revelan importantes desigualdades sociales y una tendencia a la polarización tanto en el comportamiento reproductivo como en los desempeños sociales.

El documento se estructura de la siguiente manera. En la primera sección se presenta una conceptualización de la transición a la vida adulta; en la segunda se caracteriza brevemente la maternidad adolescente en Uruguay; en la tercera se describen los datos y métodos empleados. Las secciones cuatro, cinco y seis muestran los resultados a los que se ha arribado, y la última sección brinda algunas reflexiones finales.

\section{De adolescentes a madres, complejidades para la juventud}

La adolescencia es una etapa en el curso de vida de las personas que se puede considerar como el inicio del tránsito a la vida adulta (TA). Durante esta etapa se procesan una serie de eventos ${ }^{4}$ que generan autonomía y capacidad de

4 Se consideran que los eventos que caracterizan el tránsito a la vida adulta y que se procesan a lo largo de la juventud (20 a 29 años) son: salida del sistema educativo, entrada al mercado de trabajo, formación de un hogar propio, formación de unión, llegada del primer hijo. 
autosustento e introducen a los jóvenes en la categoría de adultos (Settersten, Furstenberg y Rumbaut 2005). La transición a la vida adulta no es un proceso homogéneo entre los individuos, más bien señala diversas trayectorias que entre otras, están teñidas por la educación alcanzada, el estrato social de pertenencia y las connotaciones socio-históricas (Furstenberg et al. 2004).

La adolescencia es un constructo social relativamente reciente que se fue procesando desde mediados siglo XIX y principios del XX entre los sectores sociales medios y altos urbanos. Ello tiene diversas causas, entre las que se destaca la necesidad de considerar la niñez como etapa con derechos particulares y distintos de una etapa siguiente, diferenciándose de un estadio adulto. En este sentido emergen los conceptos de adolescencia y juventud como etapas en las que se procesan una serie de transformaciones y la preparación para el desempeño de la vida adulta (Amorín, Carril y Varela 2006; Llanes 2010; Cepal/OIJ 2004).

En los albores del siglo XXI, esa etapa intermedia entre la niñez y la juventud presenta distintas modalidades entre grupos sociales y entre sociedades, en la medida que cada una le imprime diversos significados que se corresponden con determinadas expectativas, en relación con los desempeños que se esperan para esa etapa del ciclo de vida (Dávila 2004).

Delinear cuándo se ubica la adolescencia y cuándo la juventud es una cuestión difícil de establecer, más allá de que además varía de acuerdo con cada sociedad. Durante esta etapa, los jóvenes van forjando su independencia y elaborando sus proyectos de vida que marcarán su desempeño adulto. El rango etario de la adolescencia y la juventud es variable. De acuerdo con la literatura referente para este trabajo se considera la adolescencia la etapa que transcurre entre los 10 y los 19 años y la juventud entre los 20 y los 29 años (Cepal/OIJ 2004)5.

Para determinadas sociedades, el inicio de la reproducción en la adolescencia implica experimentar un evento que se considera propio de etapas posteriores. Ello en el entendido de que, actualmente, se espera que en estas edades los jóvenes inviertan su tiempo en una mayor acumulación de activos, que al final de la juventud les posibilite un adecuada inserción en la vida adulta (Mora y De Oliveira 2009).

La maternidad en la adolescencia ${ }^{6}$, en muchos casos, está asociada a situaciones de privación social y contextos de socialización que han sido deficientes para acompañar el crecimiento y desarrollo de la adolescente en forma sólida y contenida. Las desigualdades sociales y de género y las carencias en salud sexual y reproductiva son algunos de los factores que intervienen en la maternidad a edades tempranas (Pantelides 2004; Rodríguez 2003; Stern 2004).

La trayectoria de vida de las jóvenes que cursaron su adolescencia habiendo sido madres establece, en algunos casos, dificultades en el desempeño de otros eventos - característicos de esta etapa del ciclo de vida- que preparan a las jóvenes para una sólida inserción en la vida adulta. La continuidad en el sistema educativo es una de las primeras dificultades que experimentan las jóvenes. Sin

5 Según la Organización Mundial de la Salud, entre 10 y 19 años se considera adolescente, y entre 20 y 29 años joven.

6 Se considera maternidad adolescente aquella que ocurre antes de los 20 años de edad (Cepal/OI) 2004). 
embargo, como lo revelan diferentes trabajos, el abandono escolar en muchos casos precede al embarazo, y tanto esta situación como el abandono consecuencia del hijo dificultan el reingreso a la educación (Stern y García 2001; Furstenberg 2007). Se ha debatido extensamente en relación con la vinculación entre abandono escolar, embarazo y maternidad adolescente. Aquellas jóvenes de contextos sociales críticos presentan mayor probabilidad de abandonar los estudios con anterioridad al embarazo y también se ha observado que la maternidad en la adolescencia arrastra a las jóvenes al abandono escolar (Billari y Philipov 2004; Flórez y Soto 2007; Furstenberg 2007; Rodríguez 2003).

La etapa en el curso de vida en que se opera el evento de la maternidad, constituye uno de los eventos claves que pautan el tránsito de los jóvenes a la vida adulta. Tener un hijo en la adolescencia implica asumir roles en función del estatus de madre, que conducen a adoptar en forma anticipada responsabilidades propias del ser adulto. Ello supone una entrada precaria a la vida adulta en la medida que compromete el grado de autonomía y la inserción social (Varela y Fostik 2011; Casal et al. 2006).

Adquirir el estatus de madre en la adolescencia en aquellas pertenecientes a estratos sociales vulnerables complejiza la continuidad en el sistema educativo, el acceso e inserción en el mercado de trabajo, la constitución de un hogar propio y la capacidad de ser autosuficiente. Estas limitaciones dificultan realizar una transición sólida a la adultez y traen consigo limitaciones en el proyecto de vida de las jóvenes, remitiéndolas en muchos casos al ámbito doméstico (Stern y García 2001; Alatorre y Atkin 1998). Construir un proyecto de vida diverso e insertarse en el medio social, se hace muy complejo para aquellas que fueron madres en la adolescencia (Furstenberg 1998).

En la otra margen se encuentran las jóvenes que no han experimentado la maternidad en la adolescencia y que han recorrido el proceso de transición a la adultez más tardíamente y dilatada en el tiempo. Esta situación se opera fundamentalmente en los estratos sociales medios y altos, lo que contribuye a que arriben al final de la juventud (25 a 29 años), con una mayor acumulación de activos. La literatura señala que ello se aprecia en mayores logros educativos, mejor y mayor inserción en la actividad económica, en la formación de pareja y constitución de un hogar propio, en una paridez más baja y en alcanzar mejores condiciones de vida (Alatorre y Atkin 1998; Llanes 2010).

\section{Maternidad adolescente en Uruguay}

La fecundidad y la maternidad en la adolescencia en el Uruguay es un fenómeno de larga data; sin embargo, hasta la década de 1990 no fue tenido en cuenta en el Estado, la política, la sociedad civil y la academia. En ese período, como consecuencia del elevado incremento en la tasa específica de fecundidad (10 a 14 años: 51\% y 15 a 19 años: 21\%), el fenómeno emerge para unos como preocupación de una mayor reproducción de la pobreza y para otros como indicador de importantes carencias en la salud sexual y reproductiva de las jóvenes.

En la década de 2000, la tasa de fecundidad adolescente desciende de 74 por mil (alcanzado en 1998), a 60 por mil aproximadamente, presentando un estancamiento que continúa hasta la actualidad. El nivel de la fecundidad de las 
adolescentes no acompaña el comportamiento reproductivo que han presentado las mujeres en Uruguay; este se ha caracterizado por una temprana transición demográfica en relación con la mayoría de los países latinoamericanos y actualmente presenta ciertos niveles en los indicadores de nupcialidad y fecundidad propios de la segunda transición demográfica ${ }^{7}$. Desde 2004 la fecundidad se ubica por debajo del nivel de reemplazo poblacional: actualmente la tasa global de fecundidad (TGF) es 1,93 niños por mujer (Varela et al. 2014).

Cabe destacar que el nivel de fecundidad adolescente en Uruguay se mantiene por encima del registrado a mitad del siglo pasado (53 por mil) (Varela 2009) y que está cercano al promedio de América Latina y el Caribe (70 por mil). En comparación con otras regiones del mundo, esta tasa en Uruguay aún se encuentra muy por encima del promedio de Europa (18 por mil) y América del Norte (27 por mil), superior a Asia ( 42 por mil) y algo superior al promedio del mundo $(55$ por mil) (Rodríguez y Cavenaghi 2014).

La hipótesis que subyace en relación con el estancamiento en el descenso de la fecundidad adolescente en Uruguay, se vincula entre otras con las carencias en salud sexual y reproductiva, las condiciones sociales y económicas precarias y las desigualdades de género persistentes en la sociedad. Ello se conjuga con las características propias de esta etapa evolutiva, en la que los jóvenes se sienten poco vulnerables a situaciones de riesgo, lo cual contribuye a un uso irregular de la anticoncepción, a pesar de contar con la información necesaria que no siempre implica una apropiación del conocimiento.

\section{Datos y métodos}

El estudio de los desempeños sociales diferenciales a los que arriban las jóvenes uruguayas al final de su juventud, habiendo iniciado la maternidad en la adolescencia, en etapas posteriores, o no haberla iniciado, es abordado desde el enfoque teórico-metodológico del curso de vida y bajo una modalidad principalmente exploratoria.

Las fuentes de información utilizadas son las Encuestas Nacionales de Adolescencia y Juventud realizadas en 1990 y 2008 por el Instituto Nacional de Estadística (INE). Estas encuestas son representativas del ámbito nacional urbano y abarcan, respectivamente en 1990 y $2008,6.547$ casos (entre 15 y 29 años) y 5.017 casos (entre 12 y 29 años). Estas encuestas no permiten construir las trayectorias vitales, ya que no brindan datos biográficos. Por ello, el análisis presenta limitaciones en términos explicativos acerca de las consecuencias de la maternidad temprana sobre los desempeños sociales. No se cuenta con el contexto familiar y personal de las jóvenes al momento de realizar el tránsito a la maternidad.

7 La expresión “segunda transición demográfica” fue acuñada por Dick Van de Kaa en 1986 y Ron Lesthaeghe en 1995 y hace referencia a cambios en la fecundidad, la formación y disolución de las uniones, la durabilidad de las mismas, el incremento de la cohabitación no matrimonial, la masificación de los anticonceptivos y la disminución de la fecundidad en el resto de las edades, entre otros. La segunda transición demográfica se diferencia de la primera en la centralidad de la autonomía individual y la emancipación de la mujer como generadora de cambios en la familia y en el significado de la maternidad. 
Se estudian dos cohortes de mujeres nacidas entre 1961 y 1965 y entre 1979 y 1983 y que en 1990 y 2008 se encuentran en la última etapa de la juventud (25 y 29 años). En términos absolutos, estas representan 1.197 y 651 casos respectivamente.

Se considera adolescencia antes de 20 años, juventud temprana de 20 a 24 años y juventud tardía o última etapa de la juventud de 25 a 29 años $^{8}$. Se destaca que las jóvenes tardías encuestadas en 1990 transitaron su adolescencia entre 1976 y 1984, mientras que las encuestadas en 2008 transitaron su adolescencia entre 1994 y 2002.

El análisis descriptivo desde una perspectiva comparada de la fecundidad y la maternidad en la adolescencia toma en cuenta como variables analíticas la educación alcanzada por las jóvenes, la educación alcanzada por sus madres (como variable proxy de contextos de socialización) y la situación de bienestar económico actual (pobreza y no pobreza').

Las dos cohortes en estudio corresponden a dos generaciones del país que transitaron etapas históricas diferentes. Las mujeres de la primera generación fueron adolescentes en una década en la que el país se sumergía en una crisis económica, social y política que desembocó en una dictadura militar de doce años de duración. Cabe recordar que el proceso de alta especulación y desinterés por la producción real, culmina en 1982 con una drástica crisis económica, bajo la cual se afectan las esferas de la producción, aumenta el desempleo, la inflación y el endeudamiento externo y se generan fuertes desequilibrios de la balanza de pagos (Macadar 1992). Desde la perspectiva demográfica, estas mujeres pertenecen a la generación que vivió la revolución sexual y contraceptiva y transitan su adolescencia en el período de fuerte emigración internacional como respuesta a un modelo económico agotado y a la crisis social y política que se manifiesta particularmente con la dictadura militar con fuerte represión a los militantes políticos y sindicales (Cabella y Pellegrino 2007).

Las mujeres de la segunda generación vivieron su adolescencia en la década de 1990, en la cual la economía uruguaya en términos de desempeño del producto interno bruto registra importante crecimiento con períodos de fuerte auge. No obstante, a partir de 1999 Uruguay entra en una fase recesiva que culmina en el 2002 con otra crisis económica de magnitudes comparables a la de 1982 y que repercute, entre algunas cuestiones, en acelerar el proceso creciente de emigración internacional. Además, ellas transitan su adolescencia en una etapa de grandes cambios en la familia, donde se incrementan sustantivamente los divorcios, aumentan las uniones consensuales y los hijos por fuera del matrimonio civil (Cabella 2009).

Los resultados de este trabajo se presentan en las tres siguientes secciones. En la primera y segunda parte, se presentan análisis descriptivos de variables que dan cuenta de los desempeños y transiciones de las jóvenes, vinculados con la etapa del ciclo de vida en que iniciaron la reproducción. En la tercera sección, se

8 En el marco del enfoque del curso de vida, la adolescencia (entre 10 y 19 años) constituye una etapa de la transición a la adultez. Esta se extiende desde los 12 a los 34 años. Dada las limitaciones de la encuesta que entrevista a jóvenes hasta los 29 años, se considera la última etapa de la juventud (tardía) en el quinquenio 25 a 29 años.

9 Para este trabajo la pobreza es medida en términos absolutos, es decir, se consideran pobres los hogares con un ingreso acumulado por debajo del $50 \%$ de la media del ingreso total del país urbano. 
analiza la transición al primer hijo a través del método Kaplan Meier ${ }^{10}$ estimando la probabilidad de tener el primer hijo de acuerdo con los años de educación alcanzados por las jóvenes en la última etapa de la juventud. También se estudia la vinculación de haber experimentado o no la maternidad con otros eventos característicos de la TA (edad de salida del sistema educativo, edad de entrada al mercado laboral y edad de salida del hogar de origen) y se presenta un modelo econométrico probit ${ }^{\mathrm{H}}$ para estudiar posibles determinantes de haber iniciado la transición a la maternidad en la etapa adolescente.

\section{Caminos diversos en la trayectoria reproductiva de dos generaciones}

La consideración de la edad a la cual tener un hijo ha ido cambiando a través del tiempo en el imaginario de las mujeres. Para ciertas culturas, adquirir el estatus de ser adulto no pasa necesariamente por el hecho de ser madre. De ocupar el primer lugar el casamiento y la maternidad, pasan a tener prioridad la finalización de los estudios, la inserción en el mercado de empleo y la autosuficiencia. Esta situación plantea un rezago en la edad de inicio de la maternidad (Furstenberg et al. 2004).

En América Latina este proceso es heterogéneo en función del estrato social de pertenencia, las desigualdades de género y entre las jóvenes rurales y urbanas. En promedio, las jóvenes más educadas retrasan el inicio de la maternidad. Esta opera mayoritariamente luego de haber realizado otras transiciones como la finalización de los estudios formales y la entrada al mercado de empleo. En los estratos sociales menos educados y en condiciones de vida vulnerables, tener el primer hijo se registra a edades más tempranas (Rosero-Bixby, Castro-Martín y Martín-García 2009).

Uruguay no es ajeno a estos cambios, evidenciándose el retraso en la edad de inicio de la maternidad entre las generaciones de estudio. Un trabajo previo muestra un retraso de un año, dado que el $50 \%$ de las mujeres que estaban finalizando la juventud en 1996 alcanzaban la maternidad a los 24 años; mientras que las de 2006 lo hacían a los 25 años. Entre los sectores sociales con mayor acceso a la educación, este fenómeno es más marcado dado que al final de la década del 2000 el 50\% de las mujeres con doce años y más de educación, alcanzaba la maternidad a los 29 años (Varela, Pollero y Fostik 2008).

En el caso de las mujeres que fueron madres adolescentes para una y otra generación que transitan el final de la juventud, se observa un estancamiento en el porcentaje de quienes iniciaron la maternidad en esta etapa del curso de vida (tabla 1). Esto revela que habría un cierto grupo de mujeres que, de forma estable en las distintas generaciones, inicia la maternidad en la adolescencia.

10 serie de sujetos que experimentan cierto evento y su correspondiente serie de probabilidad (o porcentaje acumulado).

11 Es un modelo de respuesta binaria, donde el interés descansa fundamentalmente en la probabilidad de respuesta que se halla estrictamente entre cero y uno (a diferencia de los modelos de probabilidad lineal). La función a través de la cual el modelo probit convierte las variables dependientes en un rango de cero a uno es de distribución normal $(0,1)$. 
La pregunta que surge es si la maternidad en la adolescencia es un fenómeno instaurado y difícil de cambiar.

Tabla 1. Mujeres de 25 a 29 años según condición de maternidad.

Uruguay, 1990 y 2008 (\%)

\begin{tabular}{|c|c|c|c|c|}
\hline Año & No madre & $\begin{array}{c}\text { Madre } \\
\text { adolescente }\end{array}$ & Madre joven & Total \\
\hline 1990 & 33,4 & 26,1 & 40,5 & 100,0 \\
\hline 2008 & 41,6 & 25,8 & 32,6 & 100,0 \\
\hline
\end{tabular}

Fuente: elaboración propia con base en ENAJ 1990 y 2008 (INE 1990b; 2008b), Uruguay.

Algo diferente sucede con el comportamiento de las que fueron madres en la juventud y aquellas que se mantienen por fuera de la maternidad. Los datos revelan una disminución entre ambas generaciones en el porcentaje de mujeres que son madres en la juventud (de $41 \%$ a $33 \%$ ) a la vez que mayor presencia de mujeres sin hijos (de 33\% a 42\%) (tabla 1). Esto da cuenta de la postergación de la maternidad que viene aconteciendo en Uruguay y que se vincula, entre otras cuestiones, con un leve retraso en la edad de entrada a la unión ${ }^{12}$, la prolongación de la educación y el incremento en la participación de la mujer en el mercado laboral'13. Esto abona la hipótesis del retraso en el inicio de la maternidad, lo que no impide que se mantenga estable el fenómeno de la maternidad en la adolescencia.

En cuanto al nivel de la fecundidad, la paridez media acumulada ${ }^{14}$ (PMA) de las jóvenes entre 25 y 29 años se mantiene prácticamente igual entre ambas décadas. También se mantiene estable entre ambas generaciones el promedio de hijos que acumulan las jóvenes que fueron madres en la adolescencia (paridez madres adolescentes: PA), al igual que aquellas que lo fueron en la juventud (paridez madres jóvenes: $\mathrm{P})^{15}$. Sin embargo, es destacable la diferencia que se observa entre ambas respecto a la paridez alcanzada. Aquellas jóvenes que iniciaron la reproducción en la adolescencia acumulan un hijo más que aquellas que lo hicieron con 20 años y más (tabla 2).

Tabla 2. Paridez media acumulada de mujeres de 25 a 29, de madres adolescentes y madres jóvenes. Uruguay 1990 y 2008

\begin{tabular}{|c|c|c|c|}
\hline Año & PMA & PA & PJ \\
\hline 1990 & 1,3 & 2,5 & 1,6 \\
\hline 2008 & 1,1 & 2,4 & 1,5 \\
\hline
\end{tabular}

Fuente: elaboración propia con base en ENAJ 1990 y 2008 (INE 1990b; 2008b), Uruguay.

12 De acuerdo con Cabella (2009), entre 1975 y 1984 19\% de las mujeres se unieron por primera vez luego de los 24 años, mientras que entre 1985 y 2001 esta proporción aumentó a 37\%.

13 De acuerdo con datos elaborados por Ciedur, la tasa de actividad de las mujeres entre 25 y 34 años pasó de ser $65,9 \%$ en 1986 a 80,7\% en 2009.

14 Paridez media acumulada es el número medio de hijos tenidos hasta determinada edad, que en promedio acumulan las mujeres de cada cohorte de edad.

15 Para este trabajo se construyen estos dos indicadores: Paridez madres adolescentes (PA) refiere al promedio de hijos que acumulan las jóvenes entre 25 y 29 años que fueron madres en la adolescencia. Paridez madres jóvenes (PJ), refiere al promedio de hijos que acumulan las jóvenes entre 2529 años y que fueron madres con 20 años y más. 
Es probable que estas diferencias se vinculen con la edad de inicio de las relaciones sexuales y la adopción o no de una conducta de anticoncepción. Como fue planteado por Davis y Blake (1956), la edad de inicio de las relaciones sexuales, la edad a la primera unión y la adopción de métodos anticonceptivos constituyen variables fundamentales en el nivel de la fecundidad.

En cuanto al orden de nacimiento, se observa una disminución entre ambas generaciones de la paridez de orden 2 (40\% a 35\%) y orden 3 (26\% a 24\%) en las jóvenes que fueron madres adolescentes. Las parideces de orden 1 se incrementan de $18 \%$ a $24 \%$. Sin embargo, se destaca que se mantienen constantes y elevadas las parideces de orden 4 y más. Aquellas que fueron madres en la juventud también incrementan las parideces de orden 1 y disminuyen las de orden 2. Además, se destaca el bajo porcentaje de estas jóvenes con tres hijos o más (tabla 3 ).

Tabla 3. Mujeres de 25 a 29, según número de hijos tenidos vivos, Uruguay, 1990 y 2008 (\%)

\begin{tabular}{|c|c|c|c|c|}
\hline \multirow{2}{*}{$N^{0}$ de hijos } & $\begin{array}{c}\text { Madre } \\
\text { adolescente }\end{array}$ & Madre joven & $\begin{array}{c}\text { Madre } \\
\text { adolescente }\end{array}$ & Madre joven \\
\hline 1 & 17,7 & 53,2 & 24,2 & 62,5 \\
\hline 2 & 40,1 & 38,2 & 35,3 & 27,8 \\
\hline 3 & 25,7 & 7,1 & 23,9 & 9,3 \\
\hline 4 Y más & 16,4 & 1,5 & 16,7 & 0,5 \\
\hline Total & 100,0 & 100,0 & 100,0 & 100,0 \\
\hline
\end{tabular}

Fuente: elaboración propia con base en ENAJ 1990 y 2008 (INE 1990b; 2008b), Uruguay.

\section{De la adolescencia a la juventud, huellas de una maternidad temprana}

¿Es posible plantearse que el inicio temprano de la maternidad dificulta las trayectorias vitales de las personas, complejizando la posibilidad de continuar los estudios, independizarse del hogar de origen y constituir un hogar propio, insertarse en la actividad económica, autosostenerse y limitar su descendencia futura? ¿Cuáles constituyen los logros de las madres adolescentes cuando arriban a la juventud? ¿Se diferencian de aquellas que pospusieron el inicio de la reproducción?

Las diversas trayectorias vitales de las personas forman parte de un proceso a lo largo del ciclo de vida, donde el contexto de socialización primaria es uno de los aspectos que interviene sobre las mismas.

La educación de la madre es una variable que se considera como aproximación al contexto de socialización de las jóvenes. La literatura señala la relación entre la educación de la madre con los logros educativos de los hijos y el proyecto de vida en general, donde las características personales de los estudiantes también constituyen un factor clave en su trayectoria educativa (Bourdieu 1977; Melhuish et al. 2001). En este sentido, se considera relevante el nivel educativo de la madre como uno de los factores intervinientes en la construcción del mismo.

Las fuentes de información empleadas, que son las Encuestas Nacionales de la Juventud, no brindan datos biográficos. Es de destacar que la información que 
aportan sobre la educación alcanzada por la madre refiere al momento actual, lo cual presenta un inconveniente y es que no necesariamente tiene que haber sido el clima educativo en el cual se sociabilizaron estas jóvenes en el pasado. Sin embargo, para el caso de los niveles educativos bajos, se puede inferir que constituyó el contexto de socialización en que se procesó la niñez de las jóvenes.

En relación con ello, la tabla 4 revela mayor nivel educativo de las madres de las jóvenes que no iniciaron tempranamente la reproducción tanto para 1990 como para 2008. La mayoría de las jóvenes de 1990 que tuvieron un hijo en la adolescencia (91\%) tuvieron madres con un nivel educativo bajo (menos de 9 años de educación). Para las jóvenes de la generación de 2008, se observa un incremento de las que tuvieron madres con hasta 12 años de educación (de $7 \%$ a $26 \%$ ). Esta situación es aún más marcada en el grupo de jóvenes que no fueron madres adolescentes. Esto condice con el aumento de los años de estudio de las nuevas generaciones entre 1990 y 2004 (Cardozo 2008).

Como se verá más adelante, la educación de la madre constituye una variable explicativa del comportamiento reproductivo de sus hijas. Esto se observa para las dos generaciones, siendo más acentuado para la generación que cursa la última etapa de la juventud en el 2008.

Al comparar el perfil de las jóvenes que han tenido un hijo en la adolescencia, con el de aquellas que pospusieron este evento, surgen diferencias y ciertos cambios entre las generaciones. Los logros alcanzados por las primeras en cuanto a nivel educativo e inserción en el mercado de trabajo son notoriamente inferiores a las restantes jóvenes ${ }^{16}$ (tabla 4).

En efecto, quienes fueron madres antes de los 20 años en las jóvenes de 1990, casi $70 \%$ de ellas apenas alcanzaban los nueve años de estudio o menos, siendo este guarismo diez puntos inferiores en el 2008 en función de la ganancia de más años de estudio acumulados. Mientras el acceso a la educación terciaria de estas jóvenes es casi nulo en 1990 (2\%), se aprecia un incremento de la misma en el 2008 (6\% de aumento en aquellas que cuentan con hasta 12 años de educación y $3 \%$ en aquellas que alcanzan la educación terciaria).

El mayor nivel educativo alcanzado entre las cohortes en estudio - como se señala en un estudio anterior - no tiene efecto en la disminución del nivel de la fecundidad en la etapa adolescente (Varela y Fostik 2011). En Chile, sin embargo, Rodríguez (2011) ha demostrado que en los últimos 20 años las adolescentes madres presentan un mayor nivel educativo. En el 2008, el 63,7\% de las madres adolescentes tenían entre 10 y 12 años de educación, mientras que en 1985 era $28,7 \%$. Ello revela, como señala Rodríguez, que la maternidad en esa etapa de la vida es un fenómeno multidimensional donde además de la educación formal alcanzada, intervienen una diversidad de factores como las oportunidades vitales, las desigualdades de género, las políticas en salud sexual y reproductiva, la contención familiar, entre otros.

Entre las que no fueron madres en la adolescencia en 1990, un 30\% alcanzaba menos de 9 años de estudio, casi la mitad entre 9 y 12 años, y una de cada 5 accede a estudios terciarios. Se destaca para estas, el cambio en el logro educativo en la

16 Dentro de la categoría no madre adolescente se incluyen tanto aquellas jóvenes que han sido madres después de los 20 años como aquellas que aún no tienen hijos. 
Tabla 4. Perfil de las mujeres según fueron madres adolescentes o no.

Uruguay, 1990 y 2008 (\%)

\begin{tabular}{|c|c|c|c|c|}
\hline \multirow[b]{2}{*}{$\begin{array}{l}\text { Variable binaria: Madre o no } \\
\text { madre en la adolescencia }\end{array}$} & \multicolumn{2}{|c|}{1990} & \multicolumn{2}{|c|}{2008} \\
\hline & $\begin{array}{c}\text { Madre } \\
\text { adolescente }\end{array}$ & $\begin{array}{c}\text { No madre } \\
\text { adolescente }\end{array}$ & $\begin{array}{c}\text { Madre } \\
\text { adolescente }\end{array}$ & $\begin{array}{c}\text { No madre } \\
\text { adolescente }\end{array}$ \\
\hline \multicolumn{5}{|l|}{ Nivel educativo joven } \\
\hline Menos de 9 años estudio & 67,3 & 30,1 & 57,7 & 14,8 \\
\hline 9 a 12 años estudio & 31,1 & 47,3 & 37,4 & 39,1 \\
\hline 13 y más años estudio & 1,6 & 22,6 & 5,0 & 46,1 \\
\hline Total & 100,0 & 100,0 & 100,0 & 100,0 \\
\hline \multicolumn{5}{|l|}{ Formación de unión } \\
\hline No unida & 32,7 & 53,4 & 43,1 & 61,8 \\
\hline Unida & 67,3 & 46,6 & 56,9 & 38,2 \\
\hline Total & 100,0 & 100,0 & 100,0 & 100,0 \\
\hline \multicolumn{5}{|l|}{ Estado conyugal actual } \\
\hline Soltero/a & 4,7 & 32,3 & 9,2 & 42,0 \\
\hline Casado/a & 64,7 & 56,5 & 25,4 & 23,3 \\
\hline Unión libre & 20,2 & 6,8 & 30,4 & 23,8 \\
\hline Divorciado, separado o viudo & 10,4 & 4,5 & 35,0 & 10,9 \\
\hline Total & 100,0 & 100,0 & 100,0 & 100,0 \\
\hline \multicolumn{5}{|l|}{ Condición de actividad } \\
\hline Activo & 52,1 & 72,9 & 64,9 & 85,0 \\
\hline Inactivo & 47,9 & 27,1 & 35,1 & 15,0 \\
\hline Total & 100,0 & 100,0 & 100,0 & 100,0 \\
\hline \multicolumn{5}{|l|}{ Tipo de hogar } \\
\hline Monoparental & 5,3 & 7,8 & 19,4 & 12,6 \\
\hline Biparental & 66,9 & 53,0 & 54,2 & 43,7 \\
\hline Extendido biparental & 15,5 & 12,8 & 11,8 & 9,8 \\
\hline Extendido monoparental & 6,0 & 6,6 & 8,2 & 6,9 \\
\hline Compuesto & 1,4 & 1,8 & 2,3 & 1,9 \\
\hline Otros & 4,9 & 17,9 & 4,1 & 25,2 \\
\hline Total & 100,0 & 100,0 & 100,0 & 100,0 \\
\hline \multicolumn{5}{|l|}{ Condición de pobreza } \\
\hline No pobre & 58,9 & 91,8 & 56,6 & 91,8 \\
\hline Pobre & 41,1 & 8,2 & 43,4 & 8,2 \\
\hline Total & 100,0 & 100,0 & 100,0 & 100,0 \\
\hline \multicolumn{5}{|l|}{ Nivel educativo madre } \\
\hline Menos de 9 años estudio & 91,1 & 75,9 & 70,8 & 44,6 \\
\hline 9 a 12 años estudio & 7,0 & 18,6 & 26,3 & 39,6 \\
\hline 13 y más años estudio & 1,9 & 5,5 & 2,9 & 15,8 \\
\hline Total & 100,0 & 100,0 & 100,0 & 100,0 \\
\hline
\end{tabular}

Fuente: elaboración propia con base en ENAJ 1990 y 2008 (INE 1990b; 2008b), Uruguay.

generación que transita la juventud en 2008. Las que realizan estudios terciarios se aproximan a la mitad de las jóvenes y 39\% logra entre 9 y 12 años de educación.

Nuevamente, el aumento de los niveles de educación para ambos grupos de jóvenes entre generaciones puede atribuirse en parte al aumento de los logros educativos entre 1990 y 2008 alcanzados por la población en general. Según Cardozo (2008), a principios de la década de 1990 las tasas netas de asistencia a la educación media ascendían a 68,6\%, mientras que en el 2007 esta cifra asciende a $71,2 \%$. Si se desagrega por ciclos, en el caso de la educación secundaria las tasas brutas de asistencia pasaron de $83,6 \%$ a $95,6 \%$ entre 1991 y 2008 , mientras que 
para el caso de la educación media superior se mantuvieron incambiadas en torno a $68 \%$.

A pesar de observarse mayores logros educativos en unas y otras jóvenes estudiadas, es evidente la brecha educativa que hay entre aquellas que realizan la transición a la maternidad en la adolescencia y las que no lo han realizado en esa etapa o se mantienen por fuera de la misma. Dicha brecha educativa se incrementa en 2008 observándose una polarización en este sentido.

Además, un estudio anterior para Uruguay (Varela y Fostik 2011) evidencia que entre las madres adolescentes, el abandono escolar precede a la maternidad. Este trabajo también mostró que la salida del sistema educativo en la adolescencia aumenta 10 veces más el riesgo de realizar la transición a la maternidad en esa etapa de la vida. El debate en torno a la relación entre abandono escolar e inicio temprano de la maternidad ha sido desarrollado por diversos autores. Las condiciones precarias de vida vulneran la posibilidad de continuar los estudios y ello se vincula a un inicio más temprano de la reproducción. A su vez, constituye una condición circular porque limita tanto la continuidad de los estudios como el abandono de ellos (Rodríguez 2005; Pantelides 2004; Stern 2004).

En relación con esto, en 1990 en casi todos los casos, tener un hijo en la adolescencia es un evento posterior a la salida de la escuela (tabla 5). Este fenómeno si bien retrocede en la generación 2008, se mantiene de manera relevante en quienes abandonan los estudios. Se destaca el incremento en aquellas que se mantienen en el sistema educativo y salen del mismo luego de tener el hijo (de $2 \%$ a $13 \%$ ). Esto muestra que hay un grupo de mujeres que permanecen en el sistema educativo mientras se hacen cargo de su maternidad a una edad temprana; su incremento tiene relación con lo presentado supra sobre un mayor porcentaje de jóvenes que fueron madres adolescentes y que alcanzan más años de educación.

Tabla 5. Mujeres de 25 a 29 que fueron madres adolescentes, según salida del sistema educativo antes, al mismo tiempo, o después de su primer hijo. Uruguay, 1990 y 2008 (\%)

\begin{tabular}{|c|c|c|c|c|}
\hline Año & Antes & Igual & Después & Total \\
\hline 1990 & 95,8 & 1,8 & 2,4 & 100,0 \\
\hline 2008 & 76,9 & 9,6 & 13,4 & 100,0 \\
\hline
\end{tabular}

Fuente: elaboración propia con base en ENAJ 1990 y 2008 (INE 1990b; 2008b), Uruguay.

Los años de estudio alcanzados por las jóvenes que vivieron el evento del primer hijo en la adolescencia demuestran que la mayoría de ellas al final de la juventud, obtienen un bajo logro educativo. También para estas, la inserción en la actividad económica evidencia, para ambas generaciones, una brecha en relación con aquellas que aún no hicieron la transición a la maternidad o la experimentaron en la juventud. Esto es coincidente con trabajos anteriores donde el análisis del modelo Cox revela que "la transición a la maternidad no constituye un evento que aumente el riesgo de hacer la transición al mercado de trabajo en la etapa de la adolescencia" (Varela y Fostik 2011, 136).

A pesar de las consideraciones planteadas, en el 2008 se destaca mayor inserción en el mercado laboral de las jóvenes que tuvieron un embarazo 
tempranamente respecto a 1990 (52\% en 1990 y 65\% en 2008). Esta situación se puede vincular con la tendencia general del mercado de trabajo en los últimos veinte años, que ha mostrado una incorporación masiva de las mujeres a la actividad laboral. Según datos elaborados a partir de las Encuestas Continuas de Hogares (ECH) realizadas por el Instituto Nacional de Estadística (INE), en 1990 la tasa de actividad del grupo de edades de 25 a 29 ascendía a 83,5\%, cifra que fue creciendo durante las décadas siguientes para llegar a un 86,4\% en el 2008.

De la combinación de dos dimensiones como la asistencia a un centro de enseñanza y la actividad en el mercado laboral, se puede arribar a una aproximación de la reclusión en el ámbito doméstico. Aquí se puede observar que en 1990, del total de mujeres que fueron madres en su adolescencia, casi la mitad de ellas no estudian ni trabajan en su etapa de juventud tardía; mientras que en 2008 esta proporción desciende a un tercio. A pesar de la reducción constatada entre ambos años, es relevante el porcentaje de mujeres que habiendo sido madres en su adolescencia, en su juventud tardía se hallan recluidas en el ámbito doméstico y probablemente dedicadas al cuidado del hogar (tabla 4).

Si se comparan con las mujeres que no fueron madres antes de los 20 años, el porcentaje de ellas que no estudia ni trabaja es $25 \%$ en 1990 y $11 \%$ en 2008 . Si bien aquí entran en juego las tendencias ya señaladas en el mercado de trabajo y en la asistencia al sistema educativo durante 1990 y 2008 , cabe anotar que el porcentaje de mujeres que no estudian ni trabajan en 2008 respecto a 1990 se redujo en mayor proporción entre quienes no fueron madres adolescentes en relación con quienes sí lo fueron.

Tanto para las generaciones de 1990 como las de 2008, las mujeres que fueron madres adolescentes alcanzan un desempeño educativo muy bajo, que genera un efecto negativo tanto en el acceso al mercado laboral como una baja participación en el ámbito público. Tales características individuales van asociadas a condiciones de vida muy limitadas, donde persisten altos niveles de pobreza.

La incidencia de la pobreza se destaca entre las mujeres que fueron madres adolescentes, tanto para 1990 como para 2008 (41\% y 43\%); lo cual indica, junto con estudios realizados para Uruguay (Varela y Fostik 2011), que en la juventud persisten similares condiciones socioeconómicas a las de sus años de adolescentes. He aquí una de las implicaciones de una maternidad temprana, su fuerte vínculo con el círculo de pobreza y sus dificultades para salir del mismo. Esto contrasta con las condiciones de vida de las jóvenes que no realizaron el tránsito a la maternidad en la adolescencia; el 92\% de ellas viven en condiciones de no pobreza para ambas generaciones.

En relación con el estado conyugal, para la cohorte de 1990 se observa un predominio del matrimonio, tanto para las jóvenes que fueron madres en la adolescencia como para aquellas que no lo fueron (65\% y 57\%). El comportamiento cambia sustancialmente para la generación de jóvenes de 2008; en ambos casos estudiados disminuye el porcentaje de jóvenes casadas $25 \%$ y 23\%). En las dos generaciones también se observa un cambio en cuanto a la unión libre; esta se incrementa y supera a las casadas tanto para aquellas que transitaron la maternidad en la adolescencia como para quienes no lo hicieron (30\% y 24\% respectivamente). En relación con el divorcio y la separación, se 
observa un incremento importante en las jóvenes que fueron madres adolescentes (10\% a 35\%). En las restantes jóvenes el aumento es menor y se aprecia un aumento del estado de soltería (32\% a 42\%). Se destaca que el estado conyugal refiere a la situación actual y que puede no haber sido el que tuvieron en la etapa adolescente (tabla 4).

Estos datos revelan los cambios que suceden en Uruguay en torno a la formación de uniones y la constitución de la familia y que se vinculan a la segunda transición demográfica. Estos cambios forman parte de las tendencias demográficas que se viven en la sociedad uruguaya. Cabella señala que:

\begin{abstract}
El descenso de los matrimonios no ha acarreado la disminución de la formación de nuevas familias, sino una fuerte desinstitucionalización de los vínculos conyugales. En los últimos diez años se duplicó con creces tanto la proporción de hombres y mujeres en unión consensual como la proporción de personas en unión libre respecto al total de personas en algún tipo de unión $(2009,6)$.
\end{abstract}

Otra característica señalada por Cabella (2009) es el cambio en el calendario de inicio de la vida conyugal, retrasando la edad a la cual se forma la primera unión.

El tipo de hogar que constituyen las jóvenes al final de su juventud revela que hoy y ayer las mujeres que tuvieron su primer hijo en la adolescencia se encuentran en un hogar de tipo biparental a pesar de cierto descenso $67 \%$ en 1990 y $54 \%$ en 2008). Esto implicaría que están mayoritariamente en situación de pareja y por ende compartiendo con otro (que no tiene porqué ser el padre del hijo) la crianza del niño. No obstante, el porcentaje de hogares donde la mujer se queda sola con sus hijos (hogar monoparental), más que se triplicó entre 1990 y 2008 (de 5\% a 19\%). Este dato es vinculante con el elevado incremento de estas jóvenes en situación de divorciadas y separadas (10\% a 33\%) y de solteras (5\% a 9\%). Como señala Cabella:
La generalización del divorcio ha sido responsable del aumento de los hogares monoparentales; según los datos de la ECH, en 2003 el 51\% de estos hogares tenía a la cabeza a una persona divorciada o separada, una mujer en el $87 \%$ de los casos. En 1981, según esta misma fuente, las personas separadas o divorciadas sólo re- presentaban el $28,3 \%$ de los jefes de hogares monoparentales $(2009,11)$.

Esto va acompañado de una tendencia creciente en la presencia de hogares cuya jefatura del hogar recae sobre las mujeres ${ }^{17}$.

Las jóvenes tardías que no fueron madres en la adolescencia también constituyen mayoritariamente hogares biparentales y presentan un descenso entre ambas generaciones (de 53\% a 44\%). La monoparentalidad también aumenta pero en una proporción mucho menor que la observada en las jóvenes que fueron madres adolescentes. Lo que es destacable es la categoría otros, que incluye a las jóvenes que se van a vivir con pares: en 1990 era $18 \%$ y se incrementa a $25 \%$. Esto muestra el cambio con las generaciones anteriores donde

17 Dada la naturaleza de los datos, no es posible identificar si las uniones hacen referencia exclusivamente a las de orden heterosexual o si se contemplan arreglos homo-parentales. 
la salida del hogar de origen era habitual por la concreción de la pareja en el matrimonio.

\section{Sustentos de la maternidad temprana}

Como hemos visto, las jóvenes tardías que fueron madres adolescentes - según lo observado en el apartado anterior- presentan diversas carencias y dificultades respecto a la educación alcanzada, las condiciones de vida, la inserción en el mercado de trabajo y se encuentran mayoritariamente recluidas en el ámbito doméstico.

Para complementar el análisis y en busca de los factores que expliquen el inicio de la maternidad en la etapa adolescente y los diferentes desempeños a los que arriban las jóvenes al final de la juventud habiendo experimentado o no el evento del primer hijo en la adolescencia, se indaga el calendario e intensidad del evento de tener el primer hijo, utilizando el método de Kaplan Meier de acuerdo con diferentes características y un modelo econométrico probit.

El análisis comparado de las curvas de supervivencia constata una brecha importante respecto a la edad de inicio de la maternidad según años de estudio, a la vez que un adelanto en el 2008 en aquellas con menos años de educación (menos de 9 años de estudio) y un retraso entre las generaciones con mayor educación (13 años y más). Esto demuestra una divergencia en el comportamiento reproductivo que refuerza las brechas existentes (gráfico 1).

Como se observa en el gráfico l, mientras que en 1990 entre las jóvenes de menor educación, 44\% ya eran madres a los 19 años, en 2008 ello se incrementa a 57\%. Por otra parte, se observa que estas jóvenes a los 29 años de edad, en su amplia mayoría ya realizaron el tránsito a la maternidad (85\% en 1990 y $89 \%$ en 2001). Entre las jóvenes tardías de mayor educación (13 años y más de estudio), no se registran jóvenes que iniciaran la reproducción hasta los 17 años, siendo casi insignificante el porcentaje acumulado de mujeres que experimentaron el evento a los 19 años (2\%). A los 29 años de edad, también se encuentran fuertes diferencias ya que en 1990 el porcentaje de jóvenes que tuvieron el primer hijo era de $24 \%$ y en 2008 de $33 \%$ (gráfico 1).

Este incremento de las jóvenes que inician la maternidad al final de la juventud, da cuenta del retraso en el inicio de la maternidad en las mujeres más educadas y que fuera mencionado anteriormente.

En suma, las curvas de supervivencia confirman las diferencias que se generan en el comportamiento reproductivo de las jóvenes, que han acumulado más años de estudio con aquellas que logran un bajo nivel educativo. A su vez, se observa una profundización de las brechas y una polarización entre las dos cohortes en estudio.

La vinculación de la maternidad en la adolescencia con los desempeños de las jóvenes respecto a otras transiciones propias de la vida adulta (como la salida del sistema educativo, la salida del hogar de origen o la entrada al mercado de trabajo) revelan y refuerzan las dificultades planteadas en relación con una sólida inserción en la vida adulta por parte de aquellas jóvenes que iniciaron la trayectoria reproductiva en la adolescencia. La transición 
Gráfica 1. Edad de inicio de la maternidad, mujeres de 25 a 29 años por años de estudio,

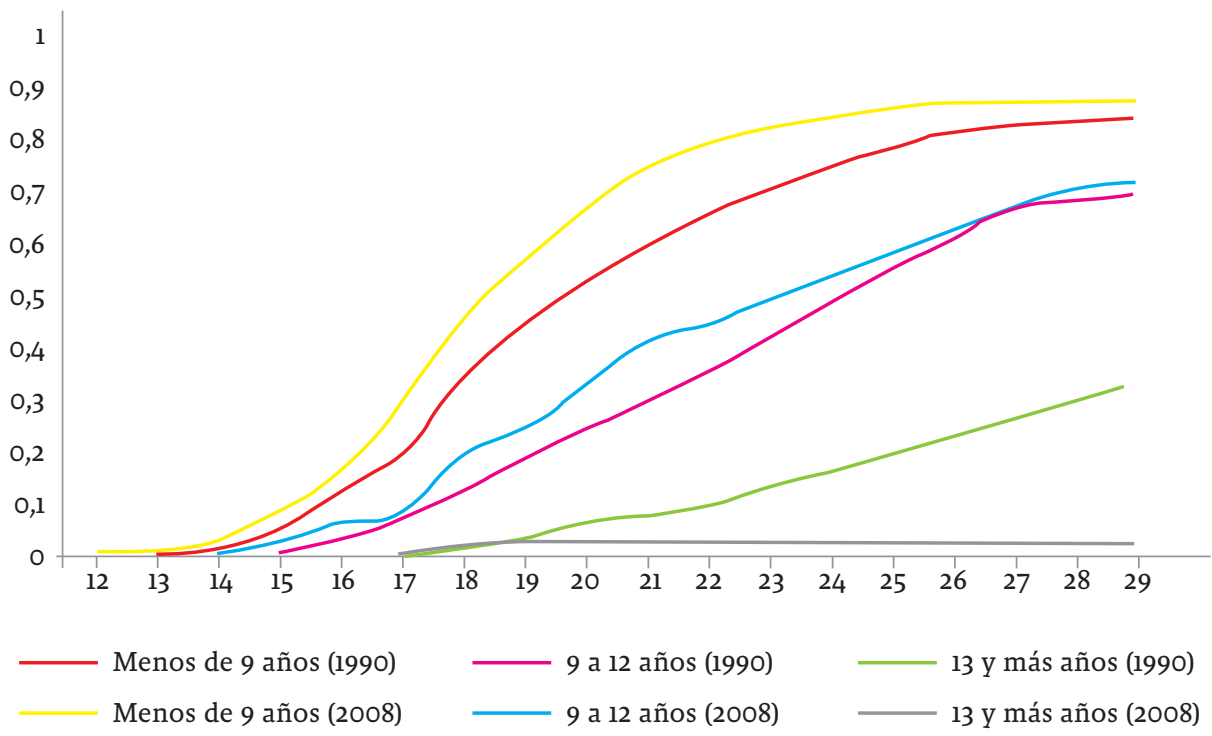

Fuente: elaboración propia con base en ENAJ y ECH (1990 y 2008) (INE 1990a; 1990b; 2008a; 2008b), Uruguay.

de salida del sistema educativo ocurre mayoritariamente con anterioridad a la maternidad o mayoritariamente en el transcurso de la adolescencia (tabla 6). En 1990 las jóvenes tardías que fueron madres en la adolescencia, el 75\% ya había salido del sistema educativo a los 15 años. La mitad de ellas a los 17 años hizo la transición al trabajo, mientras que el otro 50\% no lo ha realizado todavía en su etapa de juventud tardía. Respecto a la emancipación del hogar, la mediana de ellas la alcanzan a los 18 años y las tres cuartas partes lo hace a los 24 años. Es de destacar que una cuarta parte de estas jóvenes no ha salido del hogar.

Tabla 6. Análisis descriptivo historia de eventos (Kaplan Meier). Edad a la que experimentan eventos del curso de vida mujeres de 25 a 29 por condición de: no madre, madre en la adolescencia o juventud. Uruguay, 1990 y 2008

\begin{tabular}{|c|c|c|c|c|c|c|c|c|c|}
\hline \multicolumn{4}{|c|}{ No madre } & \multicolumn{3}{|c|}{ Madre $<20$} & \multicolumn{3}{|c|}{ Madre 20 a 29} \\
\hline 1990 & $25 \%$ & $50 \%$ & $75 \%$ & $25 \%$ & $50 \%$ & $75 \%$ & $25 \%$ & $50 \%$ & $75 \%$ \\
\hline Salida educación & 15 & 15 & 23 & 12 & 13 & 15 & 13 & 15 & 16 \\
\hline Inicio trabajo & 18 & 20 & 24 & 15 & 17 & - & 16 & 18 & 23 \\
\hline Salida hogar & 25 & - & - & 16 & 18 & 24 & 19 & 21 & 24 \\
\hline \multicolumn{10}{|l|}{2008} \\
\hline Salida educación & 20 & 26 & $\mathrm{X}$ & 13 & 15 & 17 & 15 & 18 & 23 \\
\hline Inicio trabajo & 18 & 19 & 23 & 15 & 18 & 22 & 16 & 18 & 21 \\
\hline Salida hogar & 21 & 26 & - & 16 & 18 & 20 & 18 & 20 & 23 \\
\hline
\end{tabular}

Fuente: elaboración propia con base en ENAJ 1990 y 2008 (INE 1990b; 2008b), Uruguay. 
En 2008 la transición de la educación confirma lo visualizado anteriormente respecto al incremento de los años de estudio, incluso entre las que fueron madres en la adolescencia. Lo mismo respecto a la transición al trabajo, ya que se observa mayor porcentaje de estas jóvenes que realizaron el evento de ingreso al mercado laboral en la adolescencia e incluso en la juventud. En cuanto a la edad de salida del hogar de origen, los datos muestran que logran una emancipación mayor que sus pares en 1990. El $75 \%$ de las que fueron madres en la adolescencia, a los 20 años ya había salido del hogar en relación a los 24 años en 1990.

Esta situación contrasta con el comportamiento que tuvieron las jóvenes tardías de ambas generaciones que han retrasado el inicio de la maternidad. En particular ello es aún más notorio con aquellas que en esta etapa del ciclo de vida aún se mantienen por fuera de la maternidad.

En 1990 la salida de la educación la realizan a edades más avanzadas que las que fueron madres en la adolescencia; alcanzan la mediana 2 años más tarde, y $75 \%$ la alcanzan 8 años después. La entrada al mercado de trabajo, por un lado lo postergan en las edades tempranas de la adolescencia pero luego en la juventud se insertan en mayor proporción. La salida del hogar de origen la postergan mucho más. Se destaca incluso que entre aquellas que todavía no habían sido madres solo la cuarta parte se había emancipado a los 25 años y $75 \%$ todavía no lo ha realizado. Posponer las transiciones para edades más tardías posibilita una transición a la vida adulta más sólida, lo que probablemente redunde en mejores desempeños en la adultez (tabla 6).

Por su parte, en 2008 se observa un retraso en la salida de la educación tanto para las que iniciaron la maternidad en la juventud como para aquellas que aún no lo han hecho. La transición al trabajo se adelanta en 2 años lo que puede estar influenciado por el incremento en la oferta del mercado de trabajo y la mayor preparación de los jóvenes para ingresar al mismo. También se adelanta la salida del hogar de estas jóvenes.

A modo de avanzar en la comparación y explicación del fenómeno de maternidad adolescente que experimentaron dos generaciones de mujeres uruguayas, que en 1990 y 2008 respectivamente cursaban la juventud tardía, se construye un modelo econométrico probit para analizar los determinantes de haber sido madre en la etapa adolescente.

Las variables explicativas comunes para ambos años son nivel educativo alcanzado de la madre (en tres niveles ${ }^{18}$ ), y la condición de pobreza ${ }^{19}$. Si bien esta última variable responde a la condición actual del hogar de la joven, se puede suponer que la misma no ha sufrido cambios desde su adolescencia.

Los resultados muestran que mientras más alto sea el nivel educativo de la madre de la joven, más se reducirá la probabilidad de que las hijas en su adolescencia hayan tenido el primer hijo (tabla 7). El análisis descriptivo

18 El primer nivel considerado bajo es hasta ciclo básico incompleto, el segundo nivel considerado medio es ciclo básico completo hasta bachillerato completo, y el tercer nivel considerado alto es terciaria y más.

19 La totalidad de las variables seleccionadas resultaron significativas a un $1 \%$ y a $5 \%$ para explicar el hecho de que la joven tardía haya sido madre adolescente. Los signos de las variables coinciden con los esperados a priori según la literatura. 
mostraba que en la mayoría de las jóvenes que fueron madres durante su adolescencia, sus madres tenían bajo nivel educativo. El modelo revela que esta variable es una de las que más contribuyen a explicar la maternidad en la adolescencia.

Tabla 7. Modelo econométrico probit: determinantes de la maternidad adolescente Variable dependiente binaria: mujer 25-29 si fue madre adolescente o no (madreadol)

\begin{tabular}{|l|r|r|}
\hline Año & 1990 & 2008 \\
\hline Nivel educativo medio de su madre & $-0,13$ & $-0,07$ \\
& $(-4,43)^{* * *}$ & $(-2,02)^{* *}$ \\
\hline Nivel educativo alto de su madre & $-0,14$ & $-0,18$ \\
& $(-3,17)^{* *}$ & $(-4,93)^{* * *}$ \\
\hline Condición de pobreza & 0,41 & 0,38 \\
& $(10,47)^{* * *}$ & $(6,82)^{* * *}$ \\
\hline Observaciones & 1.187 & 651 \\
\hline "Significativo al 10\%, **Significativo al 5\%, ${ }^{* * *}$ Significativo al 1\% \\
Estadísticos Z entre paréntesis
\end{tabular}

Fuente: elaboración propia con base en ENAJ 1990 y 2008 (INE 1990b; 2008b), Uruguay.

Sin embargo, se evidencian diferencias para los dos años de estudio. De acuerdo con la tabla 7, en 1990 la probabilidad de que una mujer fuera madre adolescente disminuye en $13 \%$ si el nivel educativo de su madre era medio, y $14 \%$ si dicho nivel era alto. En 2008 estos efectos marginales del nivel educativo de la madre sobre la probabilidad de que la mujer fuera madre adolescente es de $7 \%$ en el caso de nivel medio versus nivel bajo y de $18 \%$ en el caso de nivel alto versus nivel bajo ${ }^{20}$.

Esto significa que en 1990, el hecho de que la madre de la joven tuviera un nivel educativo medio, resulta más determinante para explicar que hubiera un riesgo de una maternidad precoz, que cuando se compara con 2008; situación contraria a la que ocurre cuando se estudia el efecto del nivel educativo alto de la madre, por lo que constata que a mayor nivel educativo de la madre menor riesgo de maternidad temprana.

Si se supone que la condición de pobreza de las mujeres de hoy no cambia respecto a la que tenían cuando transitaban por su adolescencia, también parece influir la variable socioeconómica en la probabilidad de que la mujer adulta de hoy haya sido madre tempranamente. Una mujer pobre hoy tiene $38 \%$ de probabilidad de haber sido madre adolescente respecto a quien no vive dichas condiciones, mientras que en 1990 esta probabilidad era de $41 \%$.

En suma, el nivel educativo de la madre y las condiciones socioeconómicas del hogar son variables que contribuyen a explicar el haber sido madre adolescente. Aunque se destacan diferencias entre ambas generaciones, persiste la importancia tanto de un mayor nivel educativo de la madre como el mayor nivel socioeconómico para disminuir la probabilidad de que la joven tardía haya tenido un hijo en su adolescencia.

20 Cabe recordar que en el caso de la educación de la madre, la referencia con la cual se observan los efectos marginales es nivel educativo bajo, y en el caso de la variable condición de pobreza la referencia es ser pobre. 


\section{Reflexiones finales}

Este trabajo aporta una primera aproximación acerca de las precariedades en los logros y desempeños sociales de las jóvenes que inician la maternidad en la adolescencia y las dificultades que devienen para su inserción social en la adultez. También muestra una situación marcadamente diferente para las jóvenes que posponen la maternidad para otras etapas del curso de vida. Ello ha quedado en evidencia a partir del análisis de las dos cohortes de jóvenes analizadas.

Los desempeños y logros que se observan en las jóvenes al final de la juventud y que tuvieron su primer hijo en la adolescencia, revelan dificultades que comprometen su adecuada inserción social, no solo en esta etapa del curso de vida, sino también en sus desempeños para su vida adulta. Esta situación se mantiene en ambas cohortes estudiadas con relativos cambios en las generaciones del 2008. Las jóvenes de hoy y ayer, que realizaron la transición de la maternidad en la etapa adolescente, arriban a la adultez como plantean Mora y De Oliveira (2009), con una trayectoria de exclusión social. Ello implica una precaria y vulnerable situación tanto en la vida privada como en la vida pública.

Las trayectorias vitales de estas jóvenes dejan al descubierto las "huellas" de la maternidad temprana en relación con aquellas que pospusieron la maternidad para etapas posteriores en el ciclo de vida. Estas se traducen en diferencias en el comportamiento reproductivo y en los desempeños sociales que de alguna manera comprometen las condiciones de vida a las que estarán sujetas en la adultez. En efecto, la paridez alcanzada por unas y otras presenta una diferencia de un hijo más. En el ámbito educativo, ellas se ubican en bajos niveles de educación alcanzados (mayoritariamente con educación primaria), e inferiores al de las jóvenes que pospusieron su maternidad. Se observa un comportamiento divergente entre ambas generaciones que profundiza las brechas educativas.

En cuanto a la inserción en la actividad económica, es relevante que casi la mitad de las que fueran madres adolescentes en 1990 no se encontraran insertas en el mercado de trabajo y a pesar de que ello mejora en la generación siguiente, se mantiene una brecha importante con aquellas que pospusieron la maternidad.

Como corolario de esta situación, se observa que gran parte de las mujeres que inician tempranamente la reproducción aparecen recluidas en el ámbito doméstico y en gran proporción en condiciones de vida carentes, donde persisten altos niveles de pobreza, situación bien diferenciada con las otras jóvenes.

La interrelación de la maternidad en la adolescencia con la edad en que se operan otros eventos característicos de la transición a la adultez (salida del sistema educativo, salida del hogar de origen, entrada al mercado de trabajo) sustenta las "huellas" que deja este evento. Ello compromete las condiciones con las que estas jóvenes arriban a la juventud tardía y que vulneran su inserción en la vida adulta. Al momento de realizar la transición a la maternidad, la mayoría de ellas ya habían salido del sistema educativo y solo la mitad logra insertarse en el mercado laboral y emanciparse del hogar de origen. Esta situación se plantea en la generación de 1990 y prácticamente se mantiene para la generación del 2008, salvo en la educación donde se observa una mayor retención en el sistema educativo. La situación es marcadamente diferente con las jóvenes que posponen la maternidad para otras etapas de la juventud o que incluso no experimentan este evento. 
El trabajo muestra que el nivel educativo de la madre y las condiciones socioeconómicas del hogar son variables que contribuyen a explicar el haber sido madre adolescente. Aunque se destacan diferencias entre ambas generaciones, persiste la importancia tanto de un mayor nivel educativo de la madre como el mayor nivel socioeconómico para disminuir la probabilidad de que la joven tardía haya tenido un hijo en su adolescencia.

En síntesis, este estudio constituye un primer análisis exploratorio sobre los desempeños sociales de las mujeres que inician la maternidad en la adolescencia y las diferencias que se manifiestan con aquellas que posponen este evento para otras etapas del ciclo de vida. Este revela que el inicio de la maternidad en la adolescencia dificulta las trayectorias vitales de las jóvenes, complejizando sus desempeños sociales y logros en la juventud y que, probablemente comprometan su adecuada inserción en la adultez.

Debe tenerse en cuenta que el análisis realizado está sujeto a ciertas limitaciones explicativas de la maternidad temprana sobre los desempeños sociales. Ello se debe a la fuente de datos con la que se cuenta. Esta no aporta datos biográficos que permitan tener certeza acerca del contexto familiar y personal de las jóvenes al momento de realizar el tránsito a la maternidad. No obstante, para el caso de aquellas jóvenes cuyas madres tienen pocos años acumulados de educación, es posible plantearse que sus hijas se han socializado en un contexto de bajo clima educativo y de bajo contexto socioeconómico.

Más allá de estas limitaciones, en este trabajo se ha podido avanzar sobre las implicaciones de la maternidad en la etapa adolescente para sus desempeños futuros y alcanzar algunas explicaciones sobre dicho comportamiento. Además, el trabajo abre la puerta para próximas investigaciones con fuentes de datos que permitan indagar sobre dimensiones que expliquen los significados sociales de la maternidad en la adolescencia y la diversidad de trayectorias que puedan realizar.

\section{Referencias bibliográficas}

Alatorre, Javier y Lucille Atkin. «De abuela a madre, de madre a hijos: repetición intergeneracional del embarazo adolescente y pobreza». En Familias y relaciones de género en transformación: cambios trascendentales en América Latina y el Caribe, de Beatriz Shmukler, 419-449. México: Population Council, 1998.

Amorín, David, Elina Carril y Carmen Varela. «Significados de maternidad y paternidad en adolescentes de estratos bajos y medios de Montevideo». En Reproducción biológica y social de la población uruguaya, de Alejandra López, 125-246. Montevideo: Trilce, 2006.

Billari, Francesco y Dimitier Philipov. «Education and the Transition to Motherhood: A Comparative Analysis of Western Europe». European Demographic Research Papers, $\mathrm{n}^{\circ}$ 3, 2004: 1-40.

Bourdieu, Pierre. «Cultural Reproduction and Social Reproduction». En Ideology in Education, de Jerome Karabel, y A. H. Halsey, 487-511. Nueva York: Oxford University Press, 1977.

Cabella, Wanda. «Dos décadas de transformaciones de la nupcialidad uruguaya. La convergencia hacia la segunda transición demográfica». Estudios Demográficos y Urbanos, Vol. 24, n² 2, 2009: 389-427. 
Cabella, Wanda y Adela Pellegrino. «La emigración internacional de uruguayos en el período reciente: un diagnóstico y aportes para discutir políticas». En Importante pero urgente. Políticas de población en Uruguay, de Juan Calvo y Pablo Mieres, 83-116. Montevideo: UNFPA-RUMBOS, 2007.

Cardozo, Santiago. Políticas educativas, logros y desafíos del sector en Uruguay 1990-2008. Montevideo: Cuadernos de la ENIA, 2008.

Casal, Joaquín, Maribel García, Rafael Merino y Miguel Quesada. «Aportaciones teóricas y metodológicas a la sociología de la juventud desde la perspectiva de la transición». Papers: Revista de Sociología, n 79, 2006: 21-48.

Cepal/OIJ. La juventud en Iberoamérica. Tendencias y urgencias. Santiago de Chile: Cepal, Naciones Unidas, 2004.

Dávila, Oscar. «Adolescencia y juventud: de las nociones a los abordajes». Última década, Vol. 12, n 21, 2004: 83-104.

Davis, Kingsley y Judith Blake. «Social Structure and Fertility: An Analytical Framework». Economic Development and Cultural Change, Vol. 4, n 3 (abril 1956): 211-235.

Flórez, Carmen y Victoria Soto. «Fecundidad adolescente y desigualdad en Colombia». Notas de Población, n 83, 2007: 41-74.

Furstenberg, Frank. «When Will Teenage Childbearing Become a Problem? The Implications of Western Experience for Developing Countries». Studies in Family Planning, Vol. 29, $\mathrm{n}^{\circ}$ 2, 1998: 246-253.

Furstenberg, Frank. Destinies of the Disadvantage: The Politics of Teenage Childbearing. Nueva York: Russel Sage Foundation, 2007.

Furstenberg, Frank, Sheela Kennedy, Vonnie Mcloyd, Rubén Rumbaut y Richard Jr. Sttersten. "Growing up in Harder to Do». The American Sociological Association, Vol. 3, $n^{\circ}$ 3, 2004: 33-41.

Llanes, Nathaly. La maternidad adolescente y su efecto sobre la salida de la escuela entre mujeres mexicanas: replanteamientos y consideraciones. México D.F.: Tesis de Maestría en Población y Desarrollo, Flacso, 2010.

Macadar, Luis. Restauración democrática y política económica. Uruguay 1985-1989. Montevideo: Banda Oriental, 1992.

Melhuish, Edward, Kathy Sylva, Pam Sammons, Iram Siraj-Blatchford y Brenda Taggart. Social/Behavioral and Cognitive Development at 3-4 Years in Relation to Family Background. Londres: Institute of Education/DfES, 2001.

Mora, Minor y Orlandina de Oliveira. «Los jóvenes en el inicio de la vida adulta: trayectorias, transiciones y subjetividades». Estudios sociológicos, Vol. XXVII, ${ }^{\circ}$ 79, 2009: 267-289.

Pantelides, Edith. «Aspectos sociales del embarazo y la fecundidad adolescentes en América Latina». En La fecundidad en América Latina: ¿transición o revolución?, de Cepal, 167-182. Santiago de Chile: Cepal, 2004.

Rodríguez, Jorge. «La fecundidad alta en América Latina y el Caribe: un riesgo en transición». Serie Población y Desarrollo, n 46 (octubre 2003): 7-69.

Rodríguez, Jorge. «Reproducción en la adolescencia: el caso de Chile y sus implicaciones de política». Revista de la Cepal, Vol. 86 (agosto 2005): 123-146.

Rodríguez, Jorge. «Reproducción adolescente y desigualdades: VI Encuentro Nacional de Juventud, Chile». RELAP, Año 5, nº 8 (enero-junio 2011): 87-113. 
Rodríguez, Jorge y Suzana Cavenaghi. «Maternidad adolescente en América Latina: el complejo, significativo y cambiante papel de la educación». 2014. http:// www.alapop.org/Congreso2014/DOCSFINAIS_PDF/ALAP_2014_FINAL224.pdf (último acceso: 18 de septiembre de 2014).

Rosero-Bixby, Luis, Teresa Castro-Martín y Teresa Martín-García. «Is Latin America Starting to Retreat from Early and Universal Childbearing?». Demographic Research, $\mathrm{n}^{\circ}$ 20, 2009: 169-194.

Settersten, Richard, Franck Furstenberg y Ruben Rumbaut. On The Frontier of Adulthood: Theory, Research and Public Policy. Chicago: The University of Chicago Press, 2005.

Stern, Claudio. «Vulnerabilidad social y embarazo adolescente en México». Papeles de Población, $\mathrm{n}^{\circ} 39$ (enero-marzo 2004): 129-158.

Stern, Claudio y Elizabeth García. «Hacia un nuevo enfoque en el campo del embarazo adolescente». En Sexualidad y salud reproductiva. Avances y retos para la investigación, de Claudio Stern y Juan Guillermo Figueroa, 331-358. México D.F.: El Colegio de México, 2001.

Varela, Carmen. «La fecundidad adolescente: una expresión de cambio del comportamiento reproductivo en el Uruguay». Revista Salud Problema, ${ }^{\circ} 6$ (junio 1999): 51-64.

Varela, Carmen. «Maternidad y fecundidad en el Uruguay a quince años de El Cairo». Documentos de Trabajo (Unidad Multidisciplinaria - Facultad de Ciencias Sociales), $\mathrm{n}^{\circ} 77$ (diciembre 2009): 1-30.

Varela, Carmen y Ana Fostik. «Maternidad adolescente en el Uruguay: ¿transición anticipada y precaria a la adultez?». Revista Latinoamericana de Población, $\mathrm{n}^{\circ} 8$ (enero-junio 2011): 115-140.

Varela, Carmen, Ignacio Pardo, Cecilia Lara, Mathías Nathan y Mariana Tenenbaum. La fecundidad en el Uruguay (1996-2011): desigualdad social y diferenciales en el comportamiento reproductivo. Fascículo 3. Montevideo: Trilce, 2014.

Varela, Carmen, Raquel Pollero y Ana Fostik. «La fecundidad: evolución y diferenciales en el comportamiento reproductivo". En Demografía de una sociedad en transición: la población uruguaya a inicios del siglo XXI, de Carmen Varela, 35-68. Montevideo: Trilce, 2008.

\section{Base de datos}

Instituto Nacional de Estadísticas (INE). Encuesta Continua de Hogares. Uruguay, 1990a. http://www.ine.gub.uy/microdatos/microdatosnew2008.asp\#ech (último acceso: diciembre de 2013).

Instituto Nacional de Estadísticas (INE). Encuesta Nacional de Adolescencia y Juventud. Uruguay, $1990 \mathrm{~b}$.

Instituto Nacional de Estadísticas (INE). Encuesta Continua de Hogares. Uruguay, 2008a. http://www.ine.gub.uy/microdatos/microdatosnew2008.asp\#ech (último acceso: diciembre de 2013).

Instituto Nacional de Estadísticas (INE). Encuesta Nacional de Adolescencia y Juventud. Uruguay, 2008b. 